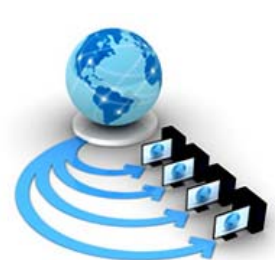

Volume 9, No. 1, January-February 2018

International Journal of Advanced Research in Computer Science

RESEARCH PAPER

\title{
DOCKING STUDIES OF STRESS TOLERANT PROTEINS WITH PROTECTIVE MOLECULES
}

\author{
Kalaiarasi,V. and Johnson, M.* \\ Centre for Plant Biotechnology, Department of Botany, \\ St. Xavier's College (Autonomous), Palayamkottai, \\ Tamil Nadu, India - 627002
}

\begin{abstract}
The present study was aimed to dock the stress tolerant proteins with the protective molecules. The stress tolerant proteins namely hexokinase, citrate synthase, phosphofructokinase from Selaginella moellendorffii, transketolase, transketolase 7 from Craterostigma plantagineum which can interact with the protective molecules trehalose sugar and growth regulating compounds IAA and tryptophan. The docking studied was performed by using the docking tool GOLD. The active sites were predicted using Q-site finder. The results were analyzed based on the binding compatibility of $\mathrm{H}$ bonds and the interactions energy was mentioned as $\mathrm{k} . \mathrm{cal} / \mathrm{mol}$.
\end{abstract}

Keywords : The results were analyzed based on the binding compatibility of $\mathrm{H}$ bonds and the interactions energy was mentioned as k.cal/mol.

\section{INTRODUCTION}

Carbohydrate plays a very important role to protect the cells due to the accumulation of sugars; their abundance was dramatically changed during dehydration and rehydration [1] The disaccharide stress response to sugar trehalose plays a key role as osmoprotectant to protect cells, leaves, protein, enzymes from drought [2-3]. Rosa and James [4], Gabriel et al., [5] studied that trehalose as the molecular chaperon protects the citrate synthase from dehydration. During desiccation the trehalose sugar perform stabilization reflects that the substitution for solvent water in $\mathrm{H}$ bonding to proteins and prevent lipid transition at membrane, and prevent the aggregation of denatured proteins and form a glass like structure around the proteins [6].

The plant growth regulator IAA present in all plants as free forms or sometimes it can bind with proteins and amino acids, when the auxin bind with the proteins it makes more rigid and stabilized the molecules [7]. IAA concentrations highly occurred during desiccation in Craterostigma wilmsii [8]. Auxin protects the plants from various abiotic stresses [9 - 12]. The auxin synthesis and transport offered the plant to adapt various stress [13]. Amino acids deposition has been observed in many plants exposed to abiotic stress [14]. Citrate synthase is an enzyme responsible for the catalyzing the reaction of the citric acid cycle the condensation of acetyl-coA and oxaloacetate to form citrate [15]. John et al., [16] observed the trehalose mediated high level of protection to the phosphofructokinase during dehydration. Phosphofructokinase involved in the regulation of glycolysis in animals [17], plants [18], insects [19], bacteria [20] and yeast [21]. Hexokinase is an enzyme converts glucose into glucose 6- phosphate by phosphorylation. Hexokinase inhibited by its own product of glucose- 6- phosphate glucose [22]. During dehydration the hexokinase activity linked to stress response sugar accumulation lead to decrease the glucose concentration at the desiccated state of Sporobolus stapfianus and X. viscosa [23]. Transketolase involved two reactions in pentose phosphate pathway, converts ribulose 5-phosphate and xylulose 5- phosphate to glyceraldehydes' 3 phosphate and sedoheptulose 7phosphate and also it converts xylulose 5- phosphate and erythrose 4 -phosphate to form glyceraldehyde 3-phosphate and fructose phosphate.

Docking is the interaction process between the orientation of target protein and ligands molecule to produce the stable compound [24]. Orientation offered the strength of association or binding affinity between the target protein and ligands molecules [24]. At present number of docking tools are available in the market. In the present study the GOLD (Genetic Optimization of Ligand Docking) based on genetic algorithm (GA) was employed for the docking of the plant compounds. Deepika et al., [25] confirmed that hydrogen bonding interactions play an important role for stability of the complex. Feruloyl CoA was docked with Cinnamoyl CoA reductase (CCR) of Leucaena leucocephala using GOLD [26]. Similarly the Aeromonas hydrophila efflux $B$ protein was docked with (EPIs) 1-(1naphthylmethyl)-piperazine (NMP) and phenyl-arginine- $\beta$ naphthylamide (PABN) [27] and STAT4 protein with Flurbiprofen [25]. Docking study was carried out IAA with lectin protein obtained from Canavalia maritima seeds [28]. With this knowledge the present study was aimed dock the following proteins namely hexokinase, citrate synthase, phosphofructokinase from Selaginella moellendorffii, transketolase, transketolase 7 from Craterostigma plantagineum with trehalose sugar, IAA and tryptophan has been studied using the dock tool GOLD.

\section{Materials and Methods}

GOLD (Genetic Optimization of Ligand Docking)

GOLD score can be calculated

Gold score $=S\left(h b \_\right.$ext $)+S\left(v d w \_e x t\right)+S\left(h b \_\right.$int $)+S$ (vdw_int)

Where $\mathrm{S}(\mathrm{hb}-\mathrm{ext})$ is the protein-ligand hydrogen bond score, S (vdw - ext) is the protein-ligand van der Waals score, S (hb-int) is the score from intermolecular hydrogen bond in the ligand and S (vdw-int) is the score from intermolecular strain in the ligand. 


\section{Active Site Prediction}

After prediction of proteins, the possible binding sites of citrate synthase phosphofructokinase, hexokinase, transketolase and transketolase 7 proteins were searched using Q- site finder (http://bmbpcu36.leeds.ac.uk/qsitefinder/).

The binding active sites were obtained for citrate synthase includes.

CYS23, GLY24, GLY25, LEU26, CYS27, VAL87, SER88, ARG89, GLY113, GLY114, ASN115, GLY116, SER117, LYS142, THR143, ILE144, ASP145, ASN146, ASP147, ILE148, LEU149, THR154, PHE157, LYS186, LEU187, MET188, ARG190, GLN191, SER192, GLY193, PHE194, ILE195, GLU244, GLY245, ALA246, GLN248, THR257, ASP258, ALA259, SER260, GLY261, ASN262, PRO263, ILE264, LEU265, GLY266, ASP267, ALA268, TYR291, ASP293, PRO294, THR295, TYR296, MET297, ILE298, ARG299, ALA300.

The binding sites were obtained for phosphofructokinase includes.

MET1, ASN2, SER26, ILE27, VAL28, SER29, ALA30, ALA31, SER32, GLY40, GLY41, SER42, GLY43, SER44, LEU45, THR46, VAL47, VAL48, ASP49, PHE58, ALA59, ILE60, SER61, ASP62, GLY63, GLY64, THR65, VAL66, ASN67, ALA68, THR69, ASP70, PHE71, LYS72, GLY89, TYR90, LEU91, ASN92, THR93, ALA94, PRO95, VAL96, ARG97, SER98, SER99, ILE100, SER101, TYR102, ILE103, ASP104, GLY105, LYS107, ILE109, LEU110, ARG111， TYR112， ARG113， GLY114, TYR115, MET134, TYR135GLY136, GLU279, HIE280, GLU281, MET282, ASN283, CYS284, ALA287, HIE291, HIE354, ARG355, VAL356, LYS358, ARG445, ARG446, SER447, HIE450, SER451, ARG452, HIE453, TRP454, TYR455, PRO465, ARG469, VAL471, ALA475

The domain sites were retrieved for hexokinase includes. ASP103, LEU104, GLY105, GLY106, THR107, ASN108, PHE109, ARG110, VAL111, GLN126, TYR128, LYS129, GLU130, VAL131, SER132, ILE133, PRO135, MET138, THR177, SER179, PHE180, PRO181, THR196, LYS197, GLY198, PHE199, ASN231, ASP232, THR233, THR236, GLY239, GLY240, ILE252, LEU253, GLY254, THR255, GLY256, SER257, ASN258, GLU317, SER321, GLY322, MET323, ARG358, THR359, PRO360, SER363, ASP455, GLY456, GLY457, LEU458, TYR459, GLU460, HIE461, TYR462, ARG488, LEU489, SER490, LYS491, ASP492, GLY493, SER494, GLY495, ILE496.

The active sites obtained for transketolase were as follows:

MET59, PHE61, ASN62, LYS64, ASN65, PRO66, TYR67, TRP68, PHE69, ARG71, ASP72, ARG73, PHE74, VAL75, LEU89, TYR94, ASP95, SER96, LYS111, PRO116, GLU117, ASN118, PHE119, GLU120, THR121, PRO122, GLY123, VAL124, GLU125, VAL126, THR127, THR128, GLN133, GLY134, SER137, ALA138, LEU141, ALA144, HIE147, LEU148, THR164, GLU321, LYS343, SER344, ILE345, ILE346, THR347, GLY348, GLU349, LEU350, LEU385, GLY387, ILE389, ALA430, ASN433, GLY434, ALA436, LEU437, HIE438, SER439, PRO440, GLY441, LEU442, VAL443, PRO444, ILE462, ALA464, LEU465, SER466, LYS467, ALA468, ARG469, VAL470, ASN502, VAL522, GLU523, ASN524, ALA525, GLY526, ARG527. The domain active site of transketolase 7 was as follows:
TYR51, MET55, PHE57, ASN58, PRO59, LYS60, ASN61, PRO62, TYR63, TRP64, PHE65, ARG67, ASP68, ARG69, PHE70, VAL71, LEU72, TYR90, ASP91, SER92, ASN114, PHE115, THR117, PRO118, GLY119, VAL120, GLU121, VAL122, SER133, HIE158, GLU317, ILE342, GLY430, ALA432, LEU433, HIE434, SER435, PRO436.

Ligands (trehalose, IAA and tyrptophan) for binding The ligands selected for this study were trehalose, indole acetic acid and tryptophan "Fig. 1, 2, 3". The interaction of trehalose (sugar joined by 1-1 alpha bond $\alpha$ D-glucopyranosyl-( $1 \rightarrow 1)-\alpha-D$-glucopyranoside. This bond makes the trehalose stable to high temperature and resist to acid hydrolysis. Trehalose form a clustering tendency due to the hydrogen bond formed between one another. This association in water to form a cluster in various sizes [29]. The ring structure Tryptophan is the precursors of IAA, IAA (indole acetic acid) or auxins are compounds with an aromatic ring and a carboxylic acid group, it generates majority of effects in plants. The 2D structure of Trehalose was drawn using ACD chemsketch (www.acdlabs.com).

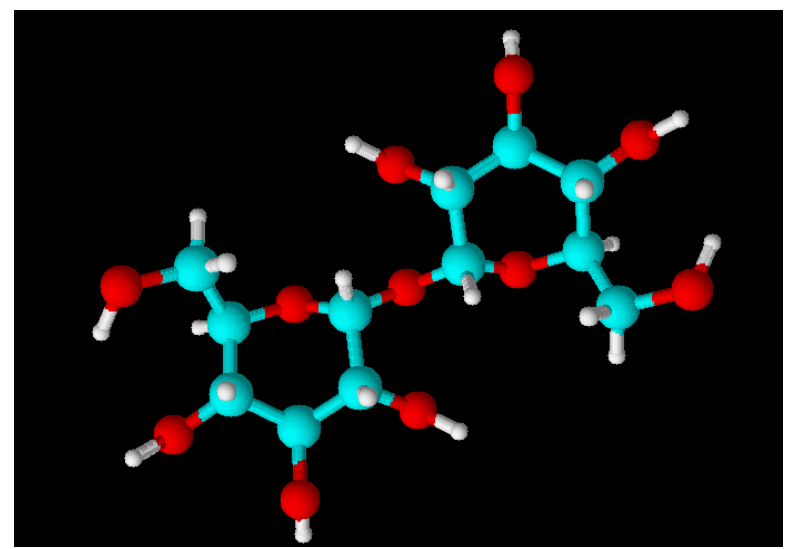

Fig.1: Structure of Trehalose

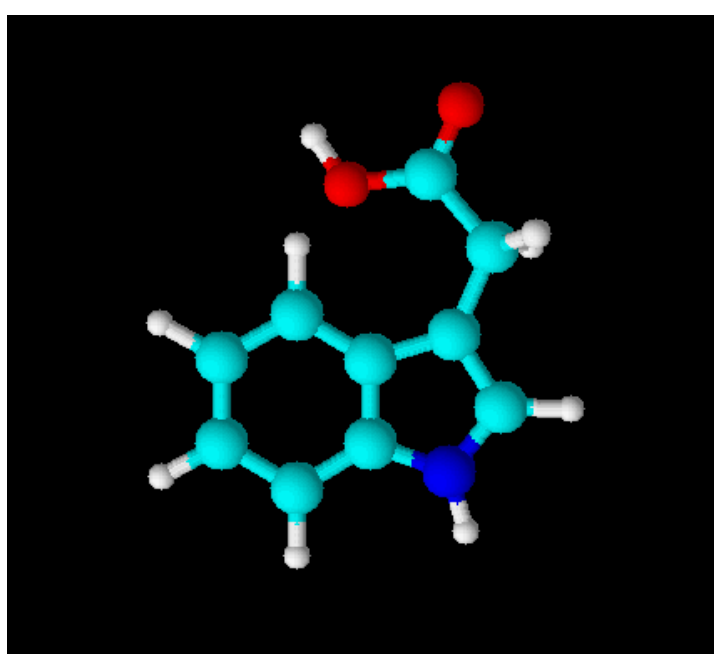

Fig. 2: Indole Acetic Acid 


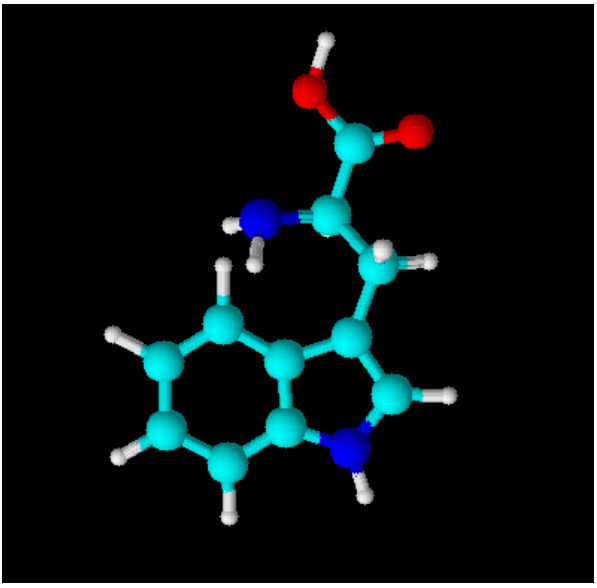

Fig. 3: Tryptophan

\section{GOLD docking simulation}

The sugar trehalose was docked with the active site of receptor citrate synthase, phosphofructokinase, hexokinase, transketolase and transketolase7 model using GOLD (Genetic Optimization of Ligand Docking WWW.CCDC.U.K) [30]. GOLD software based on genetic algorithms (GA) which expose the ligand conformational flexibility and the rational flexibility of selected receptors hydrogen. Grid was drawn for the selected proteins with the center and the size of bounding box $10 \AA$. The coordination of the closing box $(\mathrm{x}=121 \AA ; \mathrm{y}=87 \AA \mathrm{z}=45 \AA)$ were characterized from the initial set of active site residues.

\section{Results}

\section{Molecular docking}

Docking of trehalose sugar with citrate synthase, phosphofructokinase, hexokinase, transketolase and transketolase7 was carried out using GOLD software. The result of docking exposed the best fits of trehalose IAA and tryptophan with the binding receptors of proteins The results were analyzed based on the binding compatibility. Docking energy mentioned as k.cal $/ \mathrm{mol}$. 57 amino acids were active site for citrate synthase, 87 amino acids were active site for phosphofructokinase, 64 amino acids were active site for hexokinase, 80 amino acids active site for transketolase and 38 amino acids were active site for transketolase7 were observed.

The docking results of citrate synthase with trehalose six $\mathrm{H}$ bonds with the score $54.78 \mathrm{k.cal} / \mathrm{mol}$ "Fig. 4", three $\mathrm{H}$ bonds with IAA with the scores of $43.8 \mathrm{k} . \mathrm{cal} / \mathrm{mol}$ "Fig. 5", five H bonds with tryptophan with the scores of $49.63 \mathrm{k} . \mathrm{cal} / \mathrm{mol}$ "Fig.6".

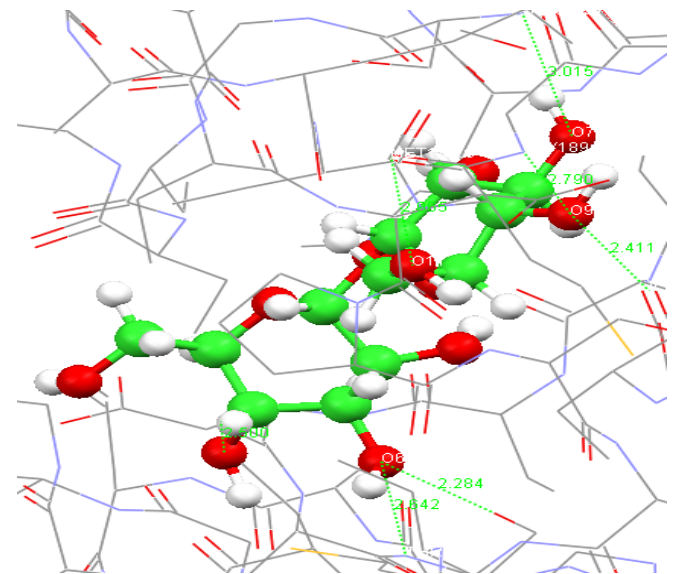

Fig. 4: Citrate synthase docking with Trehalose

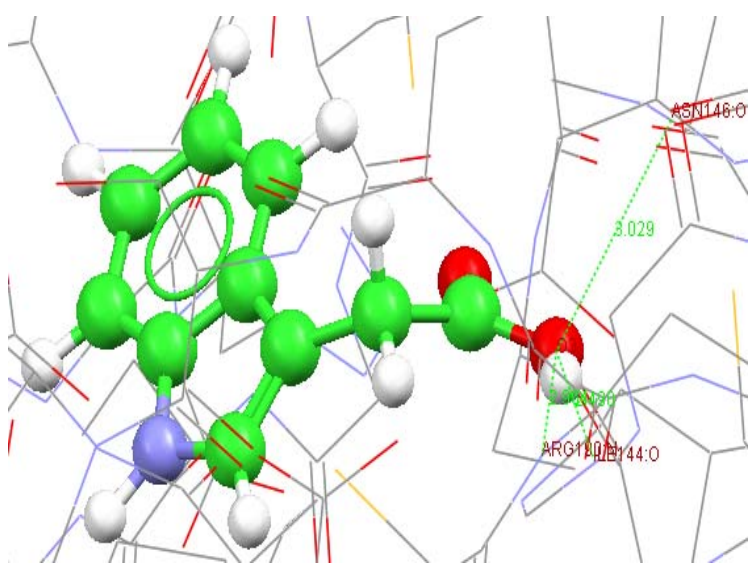

Fig .5 : Citrate synthase docking with IAA

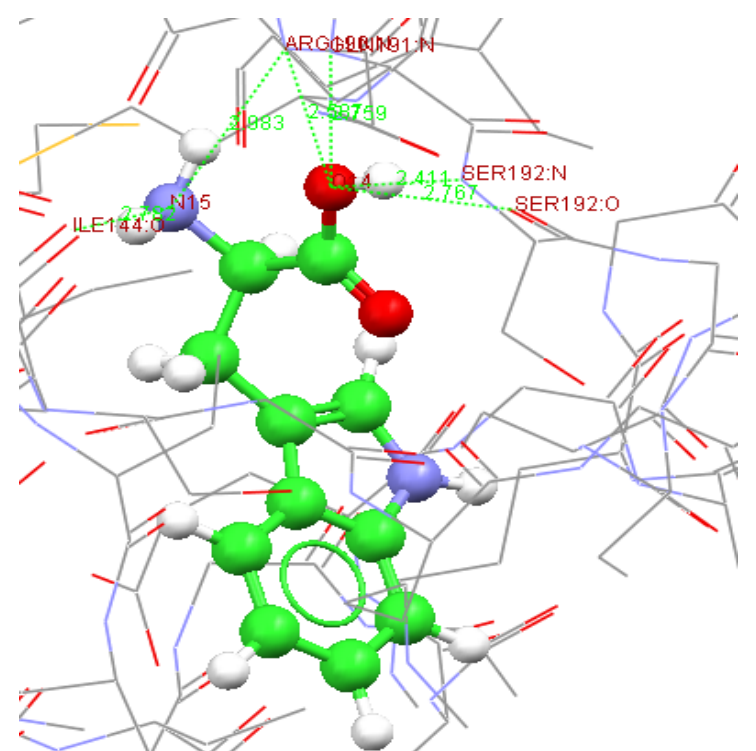

Fig. 6: Citrate synthase docking with tryptophan

Trehalose three $\mathrm{H}$ bonds with phosphofructokinase with the score of $38.78 \mathrm{k} . \mathrm{cal} / \mathrm{mol}$ "Fig.7", IAA two H bonds with PFK with the scores of $45.5 \mathrm{k} . \mathrm{cal} / \mathrm{mol}$ "Fig.8", and tryptophan four $\mathrm{H}$ bonds with PFK with the scores of 50.55 k.cal/mol "Fig.9".

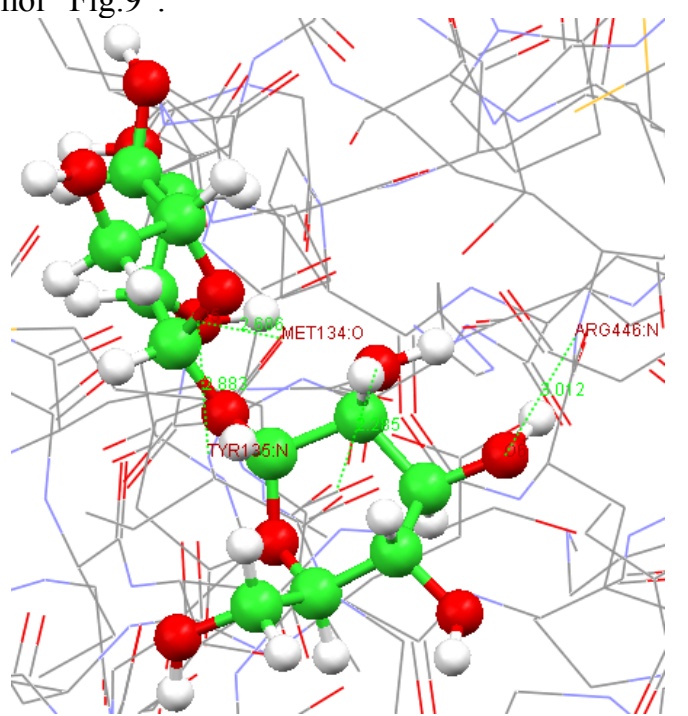

Fig. 7: phosphofructokinase docking with Trehalose 


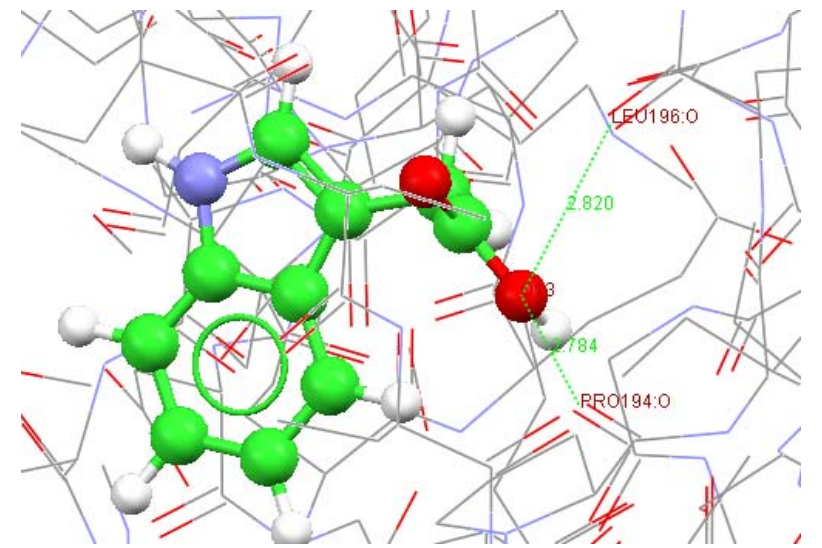

Fig. 8: Phosphofructokinase docking with IAA

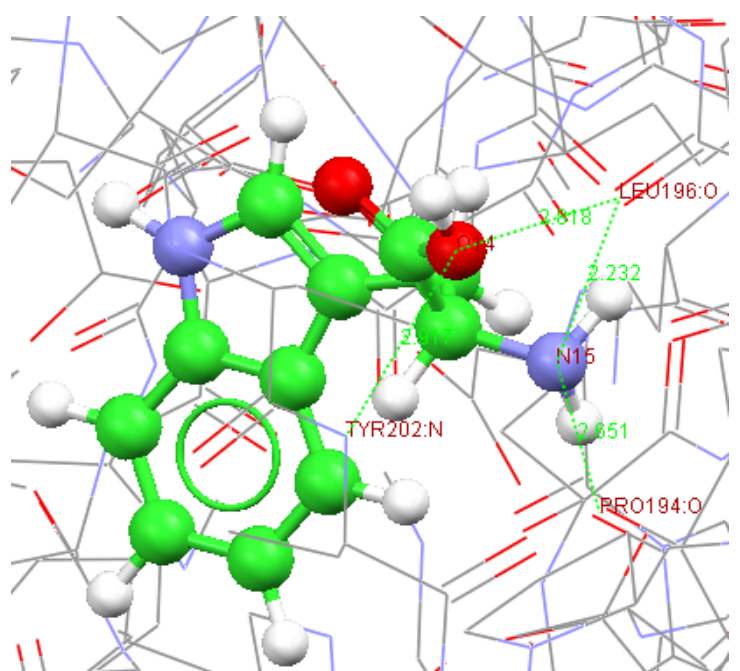

Fig. 9: Phosphofructokinase docking with tryptophan

Hexokinase docking with trehalose five $\mathrm{H}$ bonds with the score of $41.99 \mathrm{k} . \mathrm{cal} / \mathrm{mol}$ "Fig. 10", with IAA one H bond with scores of $37.5 \mathrm{k.cal} / \mathrm{mol}$ "Fig.11", with the tryptophan two $\mathrm{H}$ bonds with the scores of $42.68 \mathrm{k} . c a l / \mathrm{mol}$ "Fig.12".

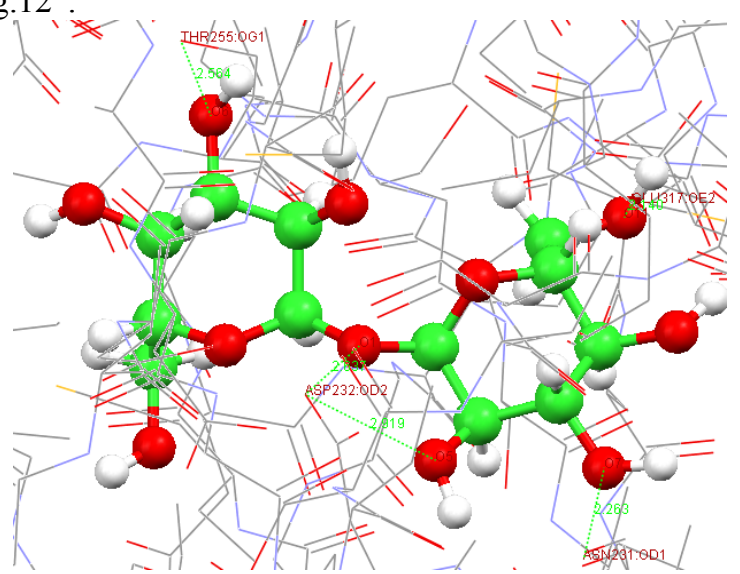

Fig. 10: Hexokinase docking with trehalose

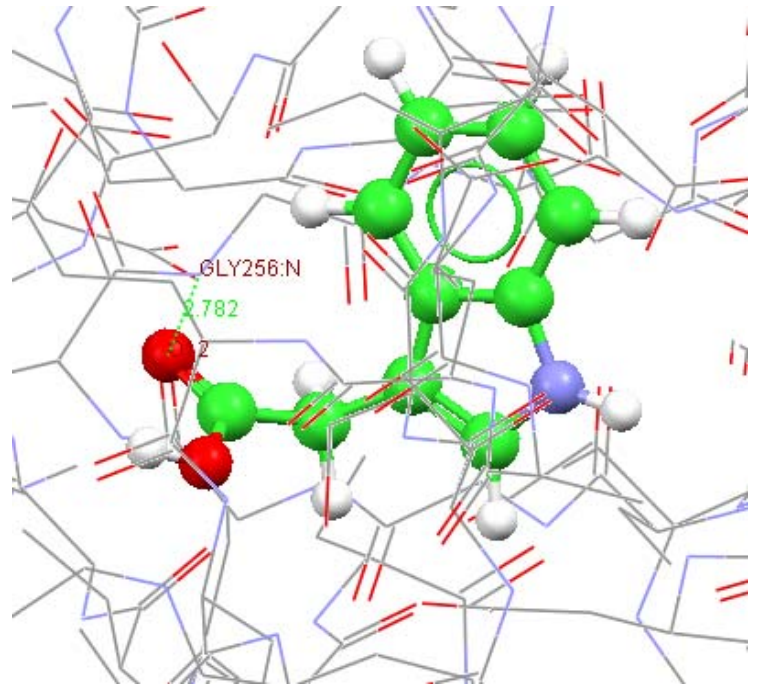

Fig. 11: Hexokinase docking with IAA

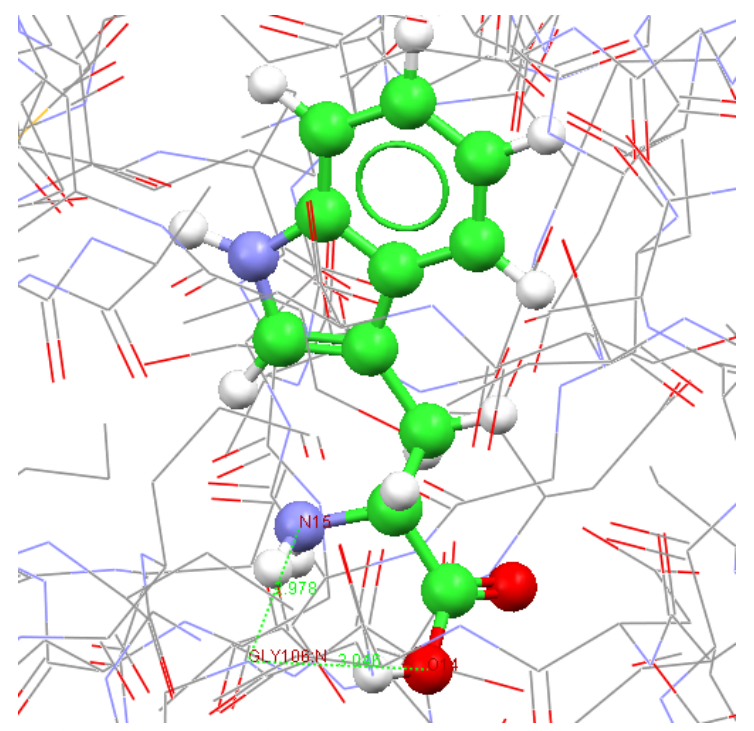

Fig. 12: Hexokianse docking with tryptophan

Trehalose two $\mathrm{H}$ bonds with transketolase with the score of $35.52 \mathrm{k.cal} / \mathrm{mol}$ "Fig.13", IAA two H bonds with transketolase with the score of $41.29 \mathrm{k.cal} / \mathrm{mol}$ "Fig.14", tryptophan three $\mathrm{H}$ bonds with transketolase with the score of $42.19 \mathrm{k.cal} / \mathrm{mol}$ "Fig. 15 ".

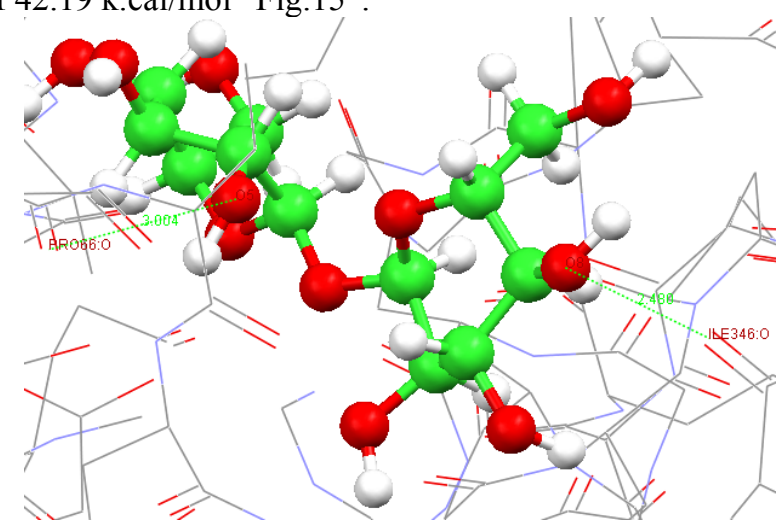

Fig. 13: Transketolase docking with trehalose 


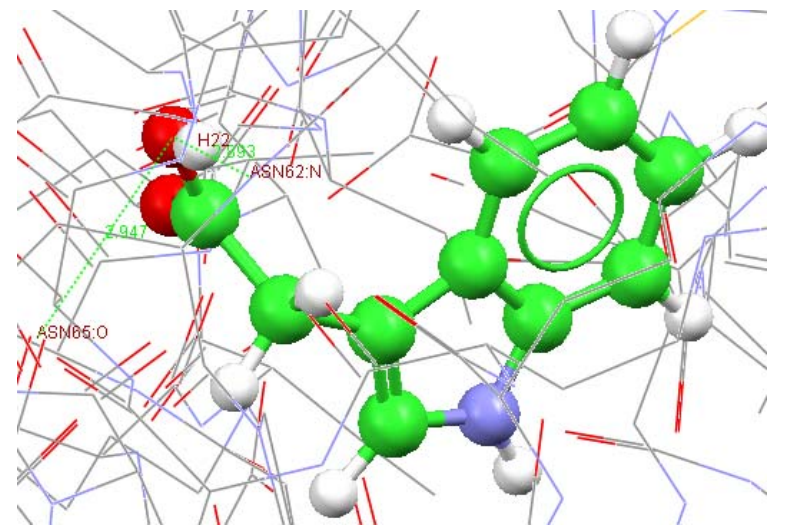

Fig. 14: Transketolase docking with IAA

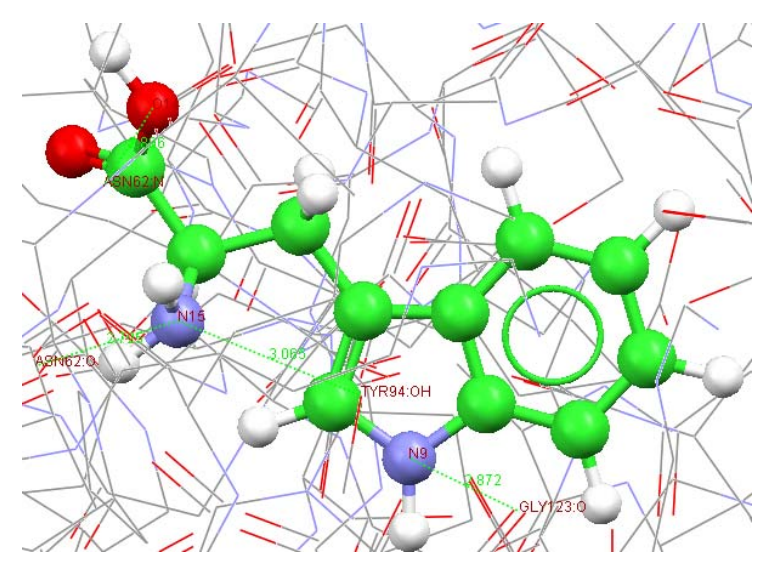

Fig. 15: Transketolase docking with Tryptophan

Transketolase 7 docking with trehalose three $\mathrm{H}$ bonding with the score of $35.34 \mathrm{k} . \mathrm{cal} / \mathrm{mol}$ "Fig.16", four $\mathrm{H}$ bonds with IAA and tryptophan with the scores of $42.26 \mathrm{k} . \mathrm{cal} / \mathrm{mol}$ "Fig.17", and 48.05 k.cal/mol "Fig.18".

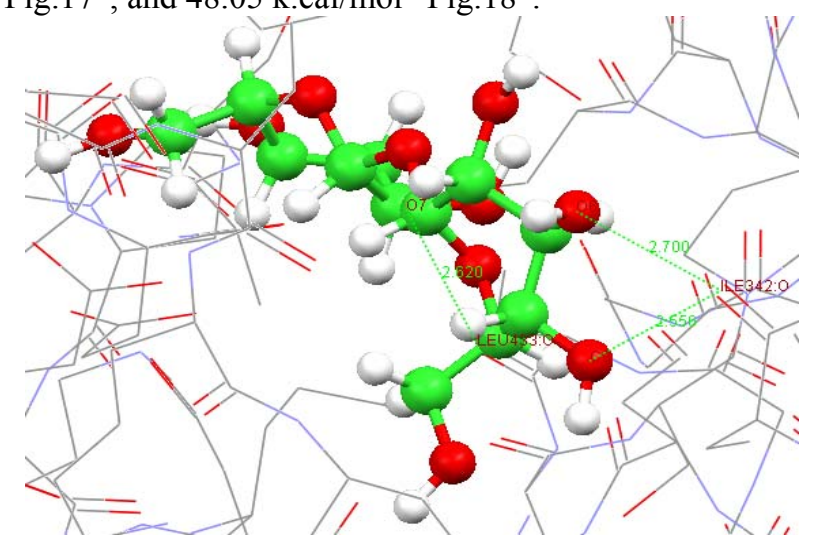

Fig. 16: Transketolase 7 docking with trehalose

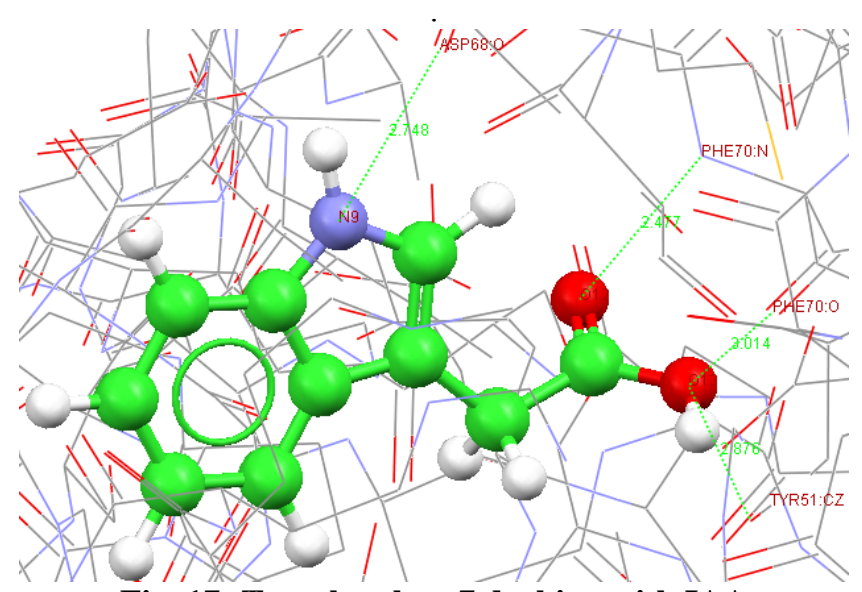

Fig. 17: Transketolase 7 docking with IAA

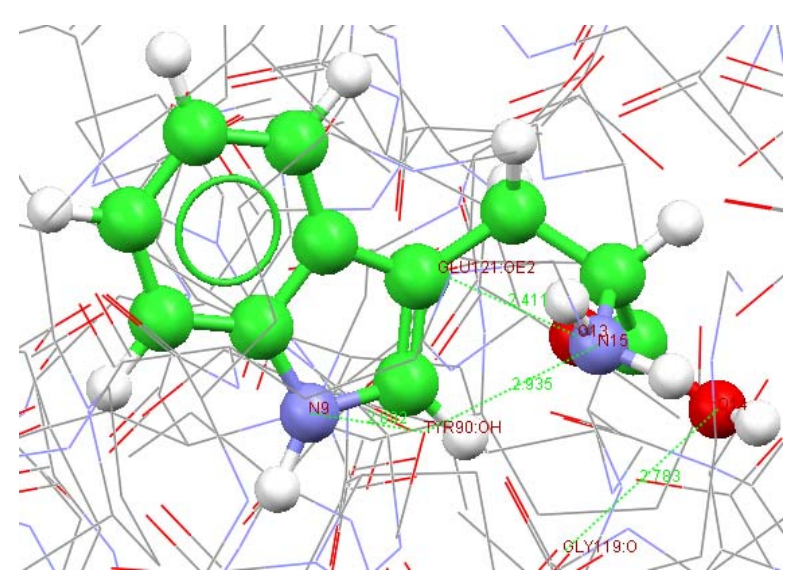

Fig. 18: Transketolase 7 docking with tryptophan

The hydrogen bond interactions and gold scores of docking conformations are depicted in Table - 1. High number of hydrogen bond interactions, it is supposed to be most active residue in interacting complex. Trehalose exhibits the highest Gold fitness with citrate synthase score of 54.78 and interacts with six hydrogen bonds and the best docking conformation showed residues SER, MET, ILE, GLY, THR. This kind of interaction may help to stabilize protein during desiccation. 
Table: 1: Docking statistics of substrates and its ligands

\begin{tabular}{|c|c|c|c|c|c|}
\hline Substrate & Ligand & Atom in protein & Atom in ligand & H-Bond Distance & $\begin{array}{l}\text { Gold } \\
\text { score }\end{array}$ \\
\hline \multirow{14}{*}{ Citrate synthase } & \multirow{6}{*}{ Trehalose } & SER192:N & $\mathrm{O} 7$ & 3.015 & \multirow{6}{*}{54.78} \\
\hline & & MET188:O & O11 & 2.965 & \\
\hline & & ILE144:O & O9 & 2.411 & \\
\hline & & GLY189:N & O9 & 2.79 & \\
\hline & & THR154:N & O6 & 2.642 & \\
\hline & & THR154:OG1 & O6 & 2.284 & \\
\hline & \multirow{3}{*}{ IAA } & ASN146:O & $\mathrm{O} 3$ & 3.029 & \multirow{3}{*}{43.8} \\
\hline & & ILE144:O & $\mathrm{O} 3$ & 2.490 & \\
\hline & & ARG190:N & $\mathrm{O} 3$ & 2.964 & \\
\hline & \multirow{5}{*}{ Tryptophan } & ARG190:N & $\mathrm{O} 1$ & 2.983 & \multirow{5}{*}{49.63} \\
\hline & & GLN191:N & $\mathrm{O} 1$ & 2.597 & \\
\hline & & SER192:N & $\mathrm{O} 1$ & 2.411 & \\
\hline & & SER192:O & $\mathrm{O} 1$ & 2.767 & \\
\hline & & ILE144:O & $\mathrm{N} 15$ & 2.782 & \\
\hline \multirow{9}{*}{ phosphofructokinase } & \multirow{3}{*}{ Trehalose } & ARG446:N & O6 & 3.012 & \multirow{3}{*}{38.78} \\
\hline & & MET134:O & O5 & 2.686 & \\
\hline & & TYR135:N & O5 & 2.883 & \\
\hline & \multirow{2}{*}{ IAA } & LEU196:O & $\mathrm{O} 13$ & 2.820 & \multirow{2}{*}{45.5} \\
\hline & & PRO194:O & $\mathrm{O} 13$ & 2.784 & \\
\hline & \multirow{4}{*}{ Tryptophan } & LEU196:O & N15 & 2.232 & \multirow{4}{*}{50.55} \\
\hline & & LEU196:O & $\mathrm{O} 14$ & 2.318 & \\
\hline & & PRO194:O & N15 & 2.651 & \\
\hline & & TR202:N & $\mathrm{N} 14$ & 2.917 & \\
\hline \multirow{8}{*}{ Hexokinase } & \multirow{5}{*}{ Trehalose } & THR255:OG1 & O6 & 2.564 & \multirow{5}{*}{41.99} \\
\hline & & ASP232:OD2 & $\mathrm{O} 1$ & 2.637 & \\
\hline & & ASP232:OD2 & O5 & 2.919 & \\
\hline & & ASN231:OD1 & $\mathrm{O} 7$ & 2.263 & \\
\hline & & GLU317:OE2 & O11 & 2.140 & \\
\hline & IAA & GLY156:N & $\mathrm{O} 12$ & 2.782 & 37.5 \\
\hline & \multirow{2}{*}{ Tryptophan } & GLY106:N & $\mathrm{N} 15$ & 2.978 & \multirow{2}{*}{42.68} \\
\hline & & GLY106:N & O1 & 3.046 & \\
\hline \multirow{7}{*}{ Transketolase } & \multirow{2}{*}{ Trehalose } & PRO66:O & O5 & 3.004 & \multirow{2}{*}{35.52} \\
\hline & & ILE346:O & O8 & 2.480 & \\
\hline & IAA & ASN65:O & $\mathrm{H} 22$ & 2.947 & 41.29 \\
\hline & IAA & ASN62:N & $\mathrm{H} 22$ & 2.892 & 41.29 \\
\hline & & GLY123:O & N9 & 2.972 & \\
\hline & Tryptophan & TYR94:OH & N15 & 3.065 & 42.19 \\
\hline & & ASN62:O & N15 & 2.235 & \\
\hline & & LEU433:O & $\mathrm{O} 7$ & 2.620 & \\
\hline & Trehalose & ILE342:O & O6 & 2.700 & 35.34 \\
\hline & & LEU433:O & O6 & 2.556 & \\
\hline & & ASP68:O & N9 & 2.748 & \\
\hline Transketolase 7 & IAA & PHE70:N & $\mathrm{O} 12$ & 2.477 & 4226 \\
\hline & 1AA & PHE70:O & $\mathrm{O} 13$ & 3.014 & \\
\hline & & TYR51:CZ & $\mathrm{O} 13$ & 2.876 & \\
\hline & Tryntonhan & GLY118:O & $\mathrm{O} 14$ & 2.703 & 48.05 \\
\hline & Iryptophan & TYR90:OH & N9 & 2.992 & 48.05 \\
\hline
\end{tabular}




\begin{tabular}{|c|c|c|c|c|} 
& \multirow{2}{*}{} & TYR90:OH & N15 & 2.935 \\
\cline { 3 - 5 } & & GLU121:OE2 & O13 & 2.411 \\
\hline
\end{tabular}

\section{DISCUSSION}

The proteins of citrate synthase, phosphofructokinase, hexokinase, transketolase and transketolase 7 were used for the docking studies with desiccation tolerant sugar trehalose, IAA and tryptophan. Trehalose is the essential components of the stress adaptive mechanisms [4]. Trehalose protects the macro molecules during desiccation [31]. Auxin regulates plant defence response and growth and structures [32-34]. When IAA interact with sugars mono and disaccharides produced low molecular compounds, as the result of IAA binds with proteins or polysaccharides produced high molecular weight compounds [35]. Tryptophan and IAA possess low binding affinity with lectin proteins Canavalia maritima seeds [29]. Branimir [7] studied the auxin binding protein and molecular modelling of auxin and antiauxin proteins. Nan [36] studied the interaction of auxin with S-adenosyl- L-homo-Cys (SAH). The interaction of sugars with citrate synthase involved the thermal protection was proved through in vitro studies [4]. The docking results explored the $\mathrm{H}$ bonding interaction of trehalose with citrate synthase and phosphofructokinase. Some of the proteins are classified as desiccation tolerance they worked both in dehydration and rehydration condition. Some of the proteins play a role of plant stress adaptations [37]. Stress induced proteins play a role to protect cellular damage involved in antioxidant defence, deposition of sugars, cell wall protection and remodelling [1]. Dehydration and heat stable enzymes occurred in plants throughout desiccation and also involved in carbohydrate metabolism, transcription factors and regulatory molecules [38-40]. Hexokinase is also a heat stable enzyme involved in carbohydrate metabolism during dehydration. Hexokinase is the very important enzyme which can be worked both in dehydration and rehydration, in desiccated state which converts all the simple sugars to stress response sugars sucrose during desiccation [23]. IAA one of the hormones which control stresses and lead to fight against defence response and plant growth response [41]. Transketolase are involved in reductive and oxidative pentose phosphate pathways and its isoforms transketolase 7 responses to synthesis octulose [42]. The genes of transketolase were identified in resurrection plant $C$. plantagineum [43]. Wang et al., [44] studied the protein transketolase response in stress tolerance in S. lepidophylla and the enzyme concentration was increased during dehydration of P. patens. Sugars protect protein through the $\mathrm{H}$ bonding to the polar amino acids in the protein [45], [46]. During interaction of sugars with phospholipids in the dried state can replace water molecules around the polar head groups [47]. Trehalose acts as the effective stabilizing molecules to protect the biological molecules against desiccation [48], [49]. Trehalose possesses unique interactions with biological systems in the dried condition which is based on water replacement hypothesis [46]. The low concentration of sugar cause to break water-water hydrogen bond and promote sugar water hydrogen bonds. The increase concentration above $25-30 \%$ of trehalose cause to increase water-water interactions lead to cause more stable and widely hydrogen bonded water clusters, these concentrations of sugars called as structure makers [50]. Trehalose, mannitol and lactose these combined sugars can preserve some enzyme proteins namely lactate dehydrogenase or phosphofructokinase through water replacements [51]. The present study revealed the interactions of stress tolerant proteins with trehalose sugar and these three stress tolerant enzymes possess weak interaction with trehalose sugar which was proven by inslico method using GOLD software.

\section{Conclusion}

The selected proteins were undergoes docking with bio molecules viz trehalose, IAA, and tryptophan through $\mathrm{H}$ bonds by GOLD software. Out of 10 results, the best one was selected based on the high score. In the docking process hydrogen bonds play a major role in structure and function of biomolecules. In this study SER 192, MET 188, ILE 144, GLY 189, THR 154, ASN 154, 146, ILE 144, ARG190, GLN191, SER192 amino acids in Citrate synthase, MET134, TYR135, ARG446, LEU196, PRO194, TR202 in Phosphofructokinase, THR255, ASP232, ASN231, GLU317, GLY156, 106 in hexokinase. POL66, ILE346, ASN65, ASN62, GLY123, TYR94, ASN62 amino acids transketolase. LEU 433, ILE 342, ASP68, PHE 70 in transketolase7 are structure interaction with ligand molecules

\section{REFERENCE}

[1]. D. Challabathula, and B. Dorothea, "Desiccation tolerance in resurrection plants: new insights from transcriptome, proteome and metabolome analysis," Front Plant Sci, vol.4,pp. 482, 2013.

[2]. W. Hartung, P. Schiller, and D.J. Karl, "Physiology of poikilohydric plants," Prog. Bot, vol.59, no. 10, pp. 299327, 1998 .

[3]. M.J Oliver, A.J Wood, and P. Mahony, "To dryness and beyond preparation for the dried state and rehydration in vegetative desiccation-tolerant plants ," Plant Growth Regul, vol. 24, pp.193-201, 1998.

[4]. I. V.Rosa, and S. C . James, "Influence of trehalose on the molecular chaperone activity of $\mathrm{p} 26$, a small heat shock/acrystallin protein," Cell Stress \& Chaperones, vol. 6, no. 2,pp. 126-135, 2001.

[5]. I. Gabriel, "The LEA proteins and trehalose loving couple: a step forward in anhydrobiotic engineering," Biochem. J, vol.410, 2008.

[6]. J. Sarvanan, D. Sourav, B. M. Asit , " Modeling the interaction of Trehalose-6-Phosphate synthase and UDPglucose bycomputational methods," Indian Journal of Bioinformatics and Biotechnology, vol. 3,no.2, pp. 55-69, 2014.

[7]. B. Branimir, K. P. Biserka, C. W. Rebecca, and T. Sanja, “ Mechanism of Auxin Interaction with Auxin Binding Protein (ABP1): A Molecular Dynamics Simulation Study Mechanism of Auxin Interaction with Auxin Binding Protein (ABP1)," A Molecular Dynamics Simulation Study Biophysical Journal, vol. 94, pp.27-37, 2008.

[8]. M. Vicre, O. Lerouxel, J. Farrant, P. Lerouge, and A. Driouich, "Composition and desiccation-induced alterations of the cell wall in the resurrection plant Craterostigma wilmsii," Physiol. Plant, vol.120, no.10, pp. 229-239, 2004. 
[9]. N.T.Bacardijeva, N.V.Christova, K. Christov, “ Reaction of peroxidase from different plant species to increased temperatures and the effect of calcium and zinc ions," In: Agriculture Eds, pp. 345-351, 1996.

[10]. P. Schopfer, A. Liszkay, M. Bechtold, G.Frahry, and A.Wagner, "Evidence that hydroxyl radicals mediate auxininduced extension growth," Planta, vol. 214, pp. 821-828, 2002.

[11]. M.J.Dragisic, J. Bogdanovic, V. Maksimovic, and M. Nikolic, "Silicon modulates the metabolism and utilization of phenolic compounds in cucumber (Cucumis sativus L.) grown at excess manganese," Journal of Plant Nutrition and Soil Sciences, vol.170, pp. 739-744, 2007.

[12]. F. Morina, L. Jovanovic, B. Kukavica , and J.S.Veljovic, "Peroxidase, phenolics and antioxidative capacity of common mullein Verbascum Thapsus L. grown in zinc excess", Archives of Biological Sciences," vol. 60, pp. 687-695, 2009

[13]. D. I. Smet, U. Voss, S. Lau, M. Wilson, N. Shao, R.E. Timme, R. Swarup, I. Kerr, C. Hodgman, R. Bock, M. Bennet, G. Jurgens, and Beeckman T, "Unraveling the evolution of Auxin signalling," Plant Physiol, vol.155, pp. 209-221, 2010.

[14]. R. Lugan, M.F.Niogret, L. Leport, J.P. Guegan, F.R. Larher, A.Savoure, A. Bouchereau, "Metabolome and water homeostasis analysis of Thellungiella salsuginea suggests that dehydration tolerance is a key response to osmotic stress in this halophyte," The Plant Journal: For Cell and Molecular Biology, vol. 64, pp. 215-229, 2010.

[15]. Roy, "Insight into sequence, structure and homology modelling of mitochondrial citrate synthase of Homo sapience," January 2015.

[16]. F.C.John, M. Beth, M.C Lois, and H.C John, "Stabilization of Phosphofructokinase during Air-Drying with Sugars and Sugar/Transition Metal Mixtures", Cryobiology, vol. 24, pp.455-464, 1987.

[17]. E.R. Stadtman, " Allosteric regulation of enzyme activity," Adv. Enzymol, vol. 28, no. 41, 1966.

[18]. D.T .Dennis, and T.P. Coultate, "Phosphofructokinase, a regulatory enzyme in plants," Biochem Biophys Res commun, vol. 25, pp. 187-191, 1966.

[19]. P. R. Walker, E. Bailey, "A comparison of the properties of the phosphofructokinases of the fat body and flight muscle of the adult male desert locust", Biochemical Journal, vol. 111 , no. 3, pp. 365-369, 1969.

[20]. J .Ferdinandus, and B. Clark, "phosphofructokinase of Arthrobacter crystallopoietes," Biochemical Journal, vol. 113, no. 4, pp. 735-736, 1969.

[21]. A. Betz, and C. Moore, "Fluctuating metabolite levels in yeast cells and extracts, and the control of phosphofructokinase activity in vitro," Arch. Biochem. Biophys, vol. 120, pp, 268-273, 1967.

[22]. R. E. Larry, Textbook of Veterinary Physiological Chemistry, Third Edition, 2015.

[23]. XXIII . A.Whittaker, A. Bochicchio, C. Vazzana, G. Lindsey, and J. Farrant, "Changes in leaf hexokinase activity and metabolite levels in response to drying in the desiccation-tolerant species Sporobo- lus stapfianus and Xerophyta viscosa", Journal of Experimental Botany, vol. 52, pp.961-969, 2001.

[24]. T. Lengauer, M. Rarey, "Computational methods for biomolecular docking”, Curr Opin Struct Biol, vol. 6, no. 3, pp. 402-6,1996.

[25]. XXV . G.Deepika, B.K.Sepideh, S.Shahab, P.Seshapani, and J.K.Pramoda, "Modeling and docking studies of signal transducers and activators of transcription 4 (stat4) protein involved in cancer", International Journal of Plant, Animal and Environmental Sciences, vol. 3, 2011.

[26]. XXVI. K. P. Nirmal, V. Vaibhav, K. Vikash, K. Ashish, P. Navneet, K. Manoj, "Structural and docking studies of
Leucaena leucocephala Cinnamoyl CoA reductase," J Mol Model, 2010

[27]. XXVII . S.Sheela, S. Jayasurya, K. B.Vadivukkarasi, and T. Florida, " Molecular modeling and docking of aheabc efflux pump in Aeromonas hydrophila", Asian Journal of Pharmaceutical and Clinical Research,vol. 6, no.1, 2013.

[28]. XXVIII. D. Plinio, C. Jose, F. Silva, A. M. R. Bruno, S.G.Tatiane, B.D.N. Raphael, A.Carlos, G. Almeida, S. D.N. Kyria, S. N. Celso, H.S. Alexandre, S. Benildo, “ Cavada Interactions between indole-3-acetic acid (IAA) with a lectin from Canavalia maritima seeds reveal a new function for lectins in plant physiology", Biochimie, vol. 95, pp. 1697- 1703, 2013.

[29]. XXVIX. S. Liel, and H. Danie,_“ "Linking Trehalose SelfAssociation with Binary Aqueous Solution Equation of State," J. Phys. Chem. B, vol. 115, no. 4, pp 624-634, 2011.

[30]. XXX. G. Jones, P. Willett, R.C. Glen, and A.R. Leach, “ Taylor R Development and validation of a genetic algorithm for flexible docking," J Mol Biol, vol. 4267, no.3, pp.727-48,1997.

[31]. XXXI. R. Zentella, G.J.O. Mascorro, P. V. Dijck, J.M. Folch, B. Bonini, and C.V. Vaeck et al, "Selaginella lepidophylla trehalose-6-phosphate synthase complements growth and stresstolerance defects in a yeast tps1 mutant", Plant Physiol,vol.119, pp. 1473-1482, 1999.

[32]. XXXII. K. Kazan, and J.M. Manners, "Linking development to defense: auxin in plant-pathogen interactions," Trends Plant Sci, vol. 14, no.7, pp.373-82, 2009.

[33]. XXXIII. S. V.M. Rivas, and J. Plasencia, "Salicylic acid beyond defence: its role in plant growth and development," J Exp Bot, vol. 62, no.10, pp. 3321-38, 2011.

[34]. XXXIV. V.B. Busov, A.M Brunner, and S.H.Strauss, “ Genes for control of plant stature and form. New Phytol," vol. 177, no.3, pp.589-607, 2008

[35]. XXXV. M.J. Ludwig, " Auxin conjugates: their role for plant development and in the evolution of land plants," J Exp Bot, vol. 62, pp.1757-1773, 2011.

[36]. XXXVI. Z. Nan, L.F. Jean, R. Jeannine, G. Ju, Y. Yue, P. Eran, P. N. Joseph, and C. Feng, " Structural, Biochemical, and Phylogenetic Analyses Suggest That Indole-3-Acetic Acid Methyl transferase Is an Evolutionarily Ancient Member of the SABATH Family," Plant Physiology, vol. 146, pp. 455-467, 2008

[37]. XXXVII. G.Q. Huang, E. Li, F.R Ge , S. Li, Q. Wang, C.Q Zhang, and Y. Zhang, "Arabidopsis RopGEF4 and RopGEF10 are important for FERONIA-mediated developmental but not environmental regulation of root hair growth," New Phytol, vol. 200,pp. 1089-1101, 2013.

[38]. XXXVIII. G.Iturriaga , M. Cushman, and J. Cushman, “ An EST catalogue from the resurrection plant Selaginella lepidophylla reveals abiotic stress-adaptive genes," Plant Biol, vol.170, pp. 1173-1184, 2006.

[39]. XXXIX. M.C.S.Rodriguez, D. Edsgärd, S.S. Hussain, D. Alquezar, M. Rasmussen, and T. Gilbert et al, "Transcriptomes of the desiccation-tolerant resurrection plant Craterostigma plantagineum," Plant J, vol.63, pp. 212-228, 2010.

[40]. XL. T.S.Gechev, M. Benina, T. Oba7]ta, T. Tohge, N. Sujeeth,and I. Minkov et al, "Molecular mechanisms of desiccation tolerance in the resurrection glacial relic Haberlea rhodopensis," Cell. Mol. Life Sci, vol. 70 pp. 689-709, 2013. B.C.Willige, M. Kutzer, F. Tebartz, and D.

[41]. XLI. G. Michael, G.B.Rebeca, L.L. Rebecca, K.G. Ian, K. Kemal, W. P. James, and S. D.Elizabeth , "Hormoneregulated defense and stress response networks contribute to heterosis in Arabidopsis F1 hybrids," PNAS Plus, pp. 6397-6406, 2015. 
[42]. XLII. B.C.Willige, M. Kutzer, F. Tebartz, and D. Bartels, "Subcellular localization and enzymatic properties of differentially expressed transketolase genes isolated from the desiccation tolerant resurrection plant Craterostigma plantagineum," Planta, vol. 229, pp. 659 - 666, 2009.

[43]. XLIII. G. Bernacchia, G. Schwall, F. Lottspeich, F. Salamini, and D. Bartels, "The transketolase gene family of the resurrection plant Craterostigma plantagineum: differential expression during the rehydration phase," EMBO J, vol. 14 pp. 610-618.1995.

[44]. XLIV. X.Wang, S.Chen, H. Zhang, L. Shi, F.Cao, and L. Guo et al, "Desiccation tolerance mechanism in resurrection fern-ally Selaginella tamariscina revealed by physiological and proteomic analysis", J. Proteome Res, vol. 9, pp. 6561-6577, 2010

[45]. XLV. J.F.Carpenter, S.J. Prestrelski, and T. Arakawa, "Separation of freezing- and drying-induced denaturation of lyophilized proteins using stress-specific stabilization. I. Enzyme activity and calorimetric studies", Arch Biochem Biophys, vol.303, pp. 456-464, 1993.

[46]. XLVI .J.F.Carpenter, and J.H. Crowe, "An infrared spectroscopic study of the interactions of carbohydrates with dried proteins," Biochemistry, vol. 28, pp. 3916-3922, 1989.
[47]. XLVII. B.P Gaber, I. Chandrasekhar, N. Pattabiraman, “" The interaction of trehalose with the phospholipid bilayer," a molecular modeling study. In: Leopold AC (ed.), Membranes, metabolism and dry organisms," pp. 231-241, Cornell University Press, 1986. Ithaca, NY.

[48]. XLVIII. G.S Jose, G. Guadalupe, P.P. Juan, and U. Salvador, "Trehalose-Mediated Protection of the Plasma Membrane $\mathrm{H}^{+}$-ATPase from Kluyveromyces lactis during Freeze-Drying and Rehydration," Cryobiology, vol. 37, no. 2, pp.131-138, 1998.

[49]. XLIX. W.Q.Sun, and P. Davidson, "Protein inactivation in amorphous sucrose and trehalose matrices: Effects of phase separation and crystallization," Bio-chim. Biophys. Acta, vol.1425, 235-244, 1998.

[50]. L. R. Giangiacomo, "Study of water-sugar interactions at increasing sugar concentration by NIR spectroscopy,"Food Chem, vol. 96, pp. 371-379, 2006.

[51]. LI. K. Kawai,and T. Suzuki, “ Stabilizing effect of four types of disaccharide on the enzymatic activity of freezedried lactate dehydrogenase: step by step evaluation from freezing to storage," Pharm Res, vol. 24, no.10, pp. 188390, 2007. 